\section{„Sustainability Transitions“ auf dem Wachstumspfad?}

\section{Bericht von der dritten internationalen Konferenz „Sustainability Transitions: Navigating Theories and Challenging Realities"}

Kopenhagen, Dänemark, 29.-31. August 2012

\section{von Ulrich Dewald, ITAS}

„Sustainability Transitions“ meint die nachhaltigkeitsorientierte Transition (im Sinne von Übergang, Überleitung, Wechsel) von sozio-technischen Systemen und basiert dabei auf verschiedenen konzeptionellen Ausgangspunkten, etwa evolutionäre Perspektiven auf technologischen Wandel oder Arbeiten zu sozio-technischen Systemen mit Anbindung an Innovationssystemtheorien. Protagonisten der „Sustainability Transitions“" erwägen zumindest auch die Gestaltung soziotechnischen Wandels, wenn man dem Titel der Konferenz folgt. Die Frage stellt sich dann, wie auf die Realität eingewirkt werden kann und ob die diskutierten Konzepte dafür geeignet sind und Instrumentarien anbieten. In solch einem gegenstandsbezogenen Forschungsprogramm werden notwendigerweise verschiedene Disziplinen berührt, was im Hinblick auf die Verständigung über Grundkonzepte sicherlich herausfordernd ist. Dies und andere Aspekte zu dieser Konferenz werden im Folgenden etwas näher betrachtet.

Die Konferenz des Sustainability Transitions Research-Netzwerks knüpfte an bereits zwei Vorgängerkonferenzen an: Nach der ersten Konferenz in Amsterdam im Jahr 2010 und nach der zweiten Konferenz letztes Jahr in Lund war nun auf den Campus der DTU (Denmarks Teknikse Universitet) beim neu formierten DIST („Center of Design and Innovation for Sustainable Transitions") eingeladen worden. Mit mehr als 300 Teilnehmern und knapp 200 Vorträgen in 42 Sitzungen setzte sich das Wachstum der Konferenzreihe auch bei der dritten Auflage fort. Dies zeigt wohl, dass hier recht erfolgreich die Bildung einer Wissenschafts-Community abläuft und dies mit hoher Anziehungskraft und Wachstumsdynamik und dabei Berührungspunkten zur und auch Gestaltungsmöglichkeiten durch TA.
Anlass genug, hier zunächst die Entstehung und disziplinäre Vorortung dieser Community kurz darzustellen und anschließend einige Eindrücke von der Konferenz wiederzugeben.

\section{Sustainable Transitions in einer heterogenen Community}

Zunächst sei ein kurzer Rückblick auf die Entstehung der transitions-Community erlaubt. Die Erstauflage der Konferenzreihe in Amsterdam deutete bereits auf den Einfluss niederländischer Wissenschaftler für den Entstehungspfad hin. Die Kernkonzepte von Nischen und Regimen in nachhaltigkeitsorientierten Transitionen gehen auf die Arbeiten von Arie Rip und René Kemp (Rip/Kemp 1998) Mitte der 1990er Jahre zurück. Darauf und auf InnovationssystemAnsätzen aufbauend wurde vor etwa zehn Jahren vom Technikhistoriker Frank Geels (SPRU Sussex) die sog. Multi-Level-Perspektive (MLP) entwickelt (Geels 2004). Mit dieser wird versucht, die Formierung technologischer Nischen in bestehenden Regimen und übergeordneten „landscapes“ konzeptionell zu rahmen. Berührungspunkte gibt es darüber hinaus auch zu dem aus der STS-Community entwickelten Konzept „Strategic Niche Management“, das einerseits deutlich einen politischen Gestaltungsanspruch (Transition Management) artikuliert, jedoch auch konzeptionelle Bausteine (Nischen) eingebracht hat. Somit liegen die Ursprünge im Wesentlichen auf Ansätzen, die ihren Ursprung in der STS-Community haben.

In vielen Präsentationen der Konferenz wurde der Rückgriff auf die MLP und ihr prägender Einfluss deutlich. Woher kommt nun die Anziehungskraft des Konzepts und der Community? Zwar setzten die Arbeiten von Geels bereits viel früher an und nahmen dabei historische Beispiele wie den Wandel von der Segelzur Dampfschifffahrt ins Auge, doch erst mit der Wahl der sich wandelnden Energieversorgung als empirischem Feld erlangte der Ansatz die jetzige Aufmerksamkeit. Die Entstehung der Scientific Community ist somit eng an ein höchst komplexes und allgegenwärtiges soziotechnisches Projekt gekoppelt, bei dem ganz klar wird, dass die bisherige Literatur zu Innovationen und 
technologischem Wandel mit ihrem Fokus auf die Entstehungsseite und einzelne Technologien eher Ausschnitte betrachtet. Hingegen ist erforderlich, die Einbettung von Technologien in sektorale Kontexte (Regime) und auch Akteure wie Nutzer von Technologien und Intermediäre zur Erklärung von Transitionen mit einzubeziehen. Der empirische Gegenstand hatte und hat immer noch eine hohe Bedeutung in der bisherigen Entwicklung der Community, auch wenn eine Ausweitung auf weitere empirische Felder stattfindet. Die MLP ermöglicht dabei ein recht einfaches Einpassen eigener empirischer Beobachtungen und Forschungsgegenstände $\mathrm{zu}$ den Wechselwirkungen neuer Technologien (Nischen) innerhalb bestehender soziotechnischer Systeme (Regime). Konzeptionelle Anziehungskraft hat dabei die evolutorische Perspektive und die Mehrebenenbetrachtung, die eben auch Blockadeverhalten und Resistenz bestehender Regime analysiert.

Dass nun konzeptionell eine Erweiterung der Community stattfindet, indem zunehmend Beiträge zu technologischen Systemen, zu räumlichen Innovationssystem-Ansätzen, zum Governance-Ansatz eingebracht werden, erfolgt aus Sicht des Berichterstatters eher über die Anziehungskraft des die Gründungsphase der Scientific Community dominierenden empirischen Gegenstands. Der Wandel der Energieversorgung, nachhaltige Lebensmittelproduktion, neue Mobilitätsformen sind Kernthemen, deren Probleme der Einbettung in eben „,challenging realities“ in der Community adressiert werden. Somit entfacht Nachhaltigkeit hier Anziehungskraft (dies übrigens, ohne dass bisher grundlegend der Begriff der Nachhaltigkeit in der transitions-community debattiert wurde) wohl auch für solche Forscher, die dies in ihren eigentlichen Heimatdisziplinen als unterbeleuchtet betrachten. Und die MLP stellt eine leicht greifbare Heuristik zur Verfügung, in die sich verschiedene empirische Objekte recht gut übertragen und mit den fachspezifischen Fragestellungen und Kernkonzepten der jeweiligen Ursprungsdisziplinen koppeln lassen (etwa die Räumlichkeit dieser Transitionen, Governance in Transitionen etc.).

Als Trend und Eindruck von der Konferenz kann jedenfalls festgehalten werden, dass eine konzeptionelle Erweiterung stattfindet, die teilweise an der Verfeinerung der bestehenden MLP ansetzt, teilweise jedoch auch darin besteht, dass zunehmend verwandte Konzepte eingebracht werden, ohne dabei einen expliziten Bezug zur MLP aufzuzeigen (was wiederum zu kritischen Nachfragen in den Sitzungen führte, womit immer noch eine Art Hegemonialstellung der MLP deutlich wurde). Dennoch wurden jedenfalls auch die spezifischen Schwächen der MLP adressiert: $\mathrm{zu}$ wenig analytisch trennscharf, $\mathrm{zu}$ historisierend und ohne prospektives Instrumentarium oder räumlich zu unsensibel sind die am stärksten vorgebrachten Kritikpunkte.

\section{Fragestellungen und Debatten}

Auf die Darstellung einzelner Konferenzbeiträge wird bei einem Umfang von fortwährend sechs parallel stattfindenden Vortragssitzungen verzichtet. $\mathrm{Zu}$ den Formaten sei nur angemerkt, dass in 90-Minuten-Sitzungen, in denen teilweise bis zu fünf Paper vorgestellt wurden, dann doch der Raum für tiefergehende Diskussionen fehlte. In drei als Plenarsitzungen organisierten Agoren wurden Themen in kurzen Statements von ausgewählten Forschern in der Community kommentiert, um diese anschließend dem Plenum zur Diskussion zu stellen. Anhand gesammelter Eindrücke aus den Vortragssitzungen und den drei Plenarsitzungen ließen sich folgende Schwerpunkte der Konferenz ausmachen:

Eine Reihe von acht Sitzungen wandte sich der Frage zu, wie Transitionsprozesse verortet sind. Etliche Beispiele zur Entstehung von Nischen, vornehmlich auf regionaler und kommunaler Ebene und im Energiebereich, wurden diskutiert. Damit kann die Geographie von Transitionen sicherlich als ein Kernthema der derzeitigen Diskussionen verstanden werden, wobei die Kritik von Geographen an der recht einfachen Analogie Nische $=$ lokal und Regime $=$ translokal nur teilweise angekommen ist. Vielfach wurden regionale Beispiele ausführlich vorgestellt, jedoch seltener die Bedeutung verschiedener Formen von Nähe für die Prozesse in den entstehenden Nischen in den Mittelpunkt gestellt. Dies hat jedoch mindestens Relevanz, wenn es um die Ableitung (technologie-)politischer Empfehlun- 
gen und damit die kontextspezifische Gestaltung von Transitionen geht.

Weiterhin zeigte sich eine zunehmende Hinwendung zur Fragestellung, wie etablierte Regime funktionieren. Hier wird ein Defizit in der bisherigen Auseinandersetzung mit Transitionen identifiziert, in der vornehmlich die neuen Technologien, also die Nischen, und die Gestaltung der Schutzräume der Nischen (,protective spaces") im Mittelpunkt standen. Zunehmend stellt sich jedoch auch die Frage, warum bestimmte Nischen sich nicht durchsetzen und sozio-technischer Wandel somit nicht stattfindet. Dies wurde in einigen Sitzungen, etwa „Strategic action of incumbent actors“ oder „Standards and translations between niche and regime" thematisiert und Beispiele für Trägheit und Barrieren in etablierten sozio-technischen Konstellationen aufgezeigt. Dies stellte zugleich auch einen Diskussionspunkt in der ersten Agora ein, in der Rob Raven (TU Eindhoven), Adrian Smith (SPRU, Sussex) und Derk Loorbach (DRIFT, Rotterdam) Stellung zu „Policy and agency of transitions“ beziehen sollten. Auch hier wurde insbesondere durch Adrian Smith der Standpunkt vorgetragen, dass die Community gut daran tue, sich nicht in den Details einzelner Nischen zu verlieren, sondern eben auch den nachhaltigen Wandel soziotechnischer Systeme, also das Skalieren der Nischen und die Funktionsweise der bestehenden Regime, in den Blick zu nehmen.

Ein weiterer verwandter Aspekt, der sowohl in den Plenarsitzungen als auch einigen Vortragssitzungen Beachtung fand, war die Frage, inwieweit dem Anspruch der Community, auch Einfluss auf Transitionen $\mathrm{zu}$ nehmen, nachgekommen werden könne. Dies erfordert auf konzeptioneller Ebene eine Einbindung solcher Ansätze, die nicht zu sehr auf Organisation und Struktur, hingegen auf Praxis und Gestaltung ausgerichtet sind. Hier regte Derk Loorbach an, die Arenen der Transitionen und die Gestaltungsfähigkeit durch einzelne Akteure in den Blick zu nehmen. Dies wurde in solchen Vortragssitzungen umgesetzt, in denen beispielsweise die Rolle von Intermediären oder die Governance von Transitionsprozessen fokussiert wurde. Inwieweit ein solcher Gestaltungsanspruch eine Lösung vom historisierenden Duktus der MLP und ihrer Makroperspektive erfordert, wird sicherlich ein zukünftiges Spielfeld für konzeptionelle Debatten bleiben.

Als weiterer Diskussionspunkt wurde der Stellenwert von Nachhaltigkeit innerhalb soziotechnischer Transitionen in der zweiten Plenarsitzung aufgegriffen. Bernhard Truffer (CIRUS, Dübendorf) stellte bewusst provokativ in den Raum, dass das konzeptionelle Instrumentarium, über das die Community bisher verfügt, keine substanziellen Aussagen zur Nachhaltigkeit im eigentlichen Sinne zulasse. Hingegen seien die Konzepte, über die bisher verfügt wird, sehr wohl geeignet um grundlegenden Mechanismen der Entstehung neuer Technologien und die Funktionsweise etablierter sozio-technischer Systeme zu analysieren. Und bereits daraus ließen sich politische Weichenstellungen ableiten. Wenn Nachhaltigkeit jedoch mehr sei als eine - sehr wohl für eine entstehende Community strategisch hilfreiche - Worthülse, dann müsste der Begriff zuvorderst auf konzeptioneller Ebene diskutiert werden, was dann zur Erweiterung des konzeptionellen Rahmens führen müsste. Lars Coenen (CIRCLE, Lund) brachte in seiner Stellungnahme dazu ein, dass zu einer Wertung in Bezug auf Nachhaltigkeit viel stärker MenschNatur-Wechselwirkungen mit berücksichtigt werden müssten, wobei die „social world“ bisher noch die Konzepte dominiere. Die Diskussion dazu setzte sich in den Flurgesprächen fort, es wurde deutlich, dass die Selbstfindung an dieser Stelle noch am Anfang steht.

\section{Zugänge für TA und Fazit}

Gerade mit einigen der zuletzt beschriebenen Diskussionspunkte bieten sich sicherlich gerade für TA als prospektiv ausgerichtetem Forschungsgebiet vielfältige Anknüpfungspunkte an die Transitions-Community. Zu den Risiken, zur Umsetzbarkeit, zu Konsequenzen zukünftiger Technologien kann ein Instrumentarium angeboten werden, das die Forschung hier stärker aus der rückblickenden Orientierung zu lösen vermag. Dabei könnte auch viel stärker die Frage der Chancen und Risiken der heute als nachhaltig betrachteten Nischen ins Zentrum gestellt werden, verbunden mit der Frage, für welche möglicherweise gerade nicht nachhaltigen Pfade gegenwärtig die Grund- 
lagen gelegt werden (Beispiel Schattenseite der Biomasse-Nutzung im Energiesystem der $\mathrm{Zu}-$ kunft). Darüber hinaus kann die TA Instrumentarien zum besseren Verständnis von Akzeptanz bzw. Nichtakzeptanz der anvisierten nachhaltigen Transitionen anbieten, mit dem auch stärker dem auf der Konferenz vielfach geäußerten Wunsch der Vertiefung von Praxis und Gestaltung von Transitionen entsprochen werden kann.

Insgesamt ermöglichte die Konferenz einen guten Überblick zum Entwicklungsstand der Community. Die gegenstandsbezogene Historie bedingt eine hohe Interdisziplinarität, die zwar dem anvisierten Gegenstand nachhaltigkeitsorientierte Transition angemessen ist, jedoch zugleich eine Verständigung über Grundbegriffe erschweren mag. Dass die MLP längerfristig den Stellenwert als konzeptionelles Gravitationszentrum zu Gunsten einer Pluralität an Konzepten abgibt, kann auch anhand der vielfach artikulierten eigenen Ansprüche der Teilnehmer, nämlich stärker Einfluss auf Gestaltung und Praxis der Transitionen zu nehmen, erwartet werden. Gerade an der Stelle ergeben sich dann auch Möglichkeiten für eine Kopplung an die TA. Jedenfalls bleibt es spannend, den bisher schwungvollen Entwicklungspfad weiterzuverfolgen, was bei der nächsten Auflage im Juni 2013 dann in Zürich möglich sein wird.

\section{Literatur}

Geels, F.W., 2004: From Sectoral Systems of Innovation to Socio-technical Systems. Insights About Dynamics and Change from Sociology and Institutional Theory. In: Research Policy 33/6-7 (2004), S. 897-920 Rip, A; Kemp, R., 1998: Technological Change. In: Rayner, S.; Malone, L. (Hg.): Human Choice and Climate Change, Vol 2: Resources and Technology. Washington, DC, S. 327-399

\section{$\langle\gg$}

\section{Training parlamentarischer TA Berichte über das PACITA-Practitioners' Meeting "Selecting the theme" sowie über die PACITA-Summer School "Renewable Energy Systems"}

Lässt sich Technikfolgenabschätzung (TA) für den parlamentarischen Beratungs- und Entscheidungsfindungsprozess trainieren? Das EU-Projekt „Parliaments and Civil Society in Technology Assessment" - kurz PACITA - geht davon aus und hat sich zum Ziel gesetzt, die Kapazitäten und institutionellen Voraussetzungen für eine wissensbasierte politische Entscheidungsfindung, v. a. zu Fragen aus Wissenschaft, technologischen Entwicklungen und Innovationen, zu erweitern. Dazu werden Methoden und Verfahren angewendet, die insbesondere demokratische, technologiepolitischeEntscheidungsfindungsprozesse unterstützen. Neben der Dokumentation verschiedener Modelle parlamentarischer TA werden die Arbeitsweisen beratender Einrichtungen in unterschiedlichen europäischen Ländern, solchen mit und solchen ohne parlamentarischer TA, zusammengetragen und vergleichend analysiert („,Documenting TA“). Im Arbeitspaket „Debating TA“ werden Debatten in Parlamenten derjenigen Länder, die bislang keine institutionalisierte parlamentarische TA haben, sowie zwei europäische TA-Konferenzen zu den Themen „Policy Areas of Great Transitions“ und „Grand Challenges" organisiert.

Um einen länderübergreifenden gesellschaftlichen Diskurs zu initiieren, werden schließlich Module entwickelt und angewandt, die TA-Praktiker und -Nutzer fortbilden sollen. Unter der Überschrift „Training TA“ geht es insbesondere darum, anhand konkreter Fragestellungen und TA-Projekte Wissenschaftler, Stakeholder und Politiker zusammenzubringen, um sich über TA-Methoden, -Erfahrungen und -Wirkungen auszutauschen. In den folgenden Beiträgen wird von zwei Veranstaltungen aus diesem Arbeitspaket berichtet: Carmen Priefer besuchte das erste von vier Practitioners ${ }^{\text {}}$ Meetings (,Selecting the theme"), das im September 2012 in Lissabon stattfand. Die nächsten Meetings werden 2013 und 2014 in drei weiteren Ländern veranstaltet, die keine parlamentarische TA haben: zu den Themen „Approaches and meth- 